\title{
Insiders and outsiders: paid contributors and the dynamics of cooperation in community led F/OS projects
}

\author{
Evangelia Berdou \\ Media and Communications Department, London School of Economics \\ and Political Science, Houghton Street, London W2A 2AE, UK \\ e.berdou@Ise.ac.uk
}

\begin{abstract}
This paper examines the role of paid developers in mature free/open source (F/OS) communities. In particular it provides a typology for their involvement based on their employment and sponsorship arrangements and elaborates a framework for understanding the dynamics of cooperation developing between them and the volunteers based on their community ties. The evidence presented is drawn from individual interviews conducted with volunteer and paid contributors from the GNOME and KDE projects within the context of a $\mathrm{PhD}$ research focusing on commercialization and peripheral participation in F/OS communities. The paper highlights the various interdependencies that form between communities and companies and adds to our understanding of the dynamics of commercialization in $\mathrm{F} / \mathrm{OS}$ projects.
\end{abstract}

\section{Introduction}

Companies contribute to F/OS projects in many ways. They support promotional activities and community conferences, including providing venues, travel costs and hardware. The website and the CVS tree of the GNOME project, for example, is hosted by Redhat and KDE's is hosted by Trolltech. However, arguably the most important and, many would say, the most potent form of involvement of companies in projects, since it has a direct impact on the development process, is through their contributing labour by committing employees to the development process.

This issue has been largely overlooked in the F/OS literature. This paper addresses this gap by providing a typology for the involvement of paid contributors in community led projects and contributes to our understanding of their involvement in terms of their community ties. The aim of the paper is to highlight certain aspects of commercialization in community led projects. The study is based on $\mathrm{PhD}$ research into the dynamics of cooperation in F/OS projects, which investigates commercialization and peripheral participation (integration of new coders and participation of non-coders).

Please use the following format when citing this chapter:

Berdou, E., 2006, in IFIP International Federation for Information Processing, Volume 203, Open Source Systems, eds. Damiani, E., Fitzgerald, B., Scacchi, W., Scotto, M., Succi, G., (Boston: Springer), pp. 201-208 


\section{Background to the study}

This section situates the argument within the context of existing contributions related to commercialization of F/OS communities and outlines the theoretical and methodological framework for the study.

\subsection{F/OS communities in/and the economy}

The considerations of commercialization issues needs to be positioned within the corpus of contributions related to commercialization of F/OS communities. F/OS communities have often been considered to occupy a space between the organized supply of products and services offered by firms and the wider, emergent, market dynamics of software production, supply and demand. O'Mahony [1], for example, talks of community managed open source projects as a new type of commercial actor and von Hippel [2] considers open source development as the template for an innovation process which is primarily driven by software users.

At the same time it has often been argued, by both practitioners and academics, that the motivations of developers contributing to F/OS projects differ significantly from those underlying proprietary software development [3-5]. This view has been interpreted as suggesting a contrast between two kinds of economic rationality; the gift economy and the exchange economy. Whereas the first is based on the principle of reciprocity, upholds the idea of software as a public good and is often associated with abundance, the second relies predominantly on monetary flows, market transactions and is organised around a scarcity of resources.

This interpretation has been challenged on several fronts by researchers who draw attention to the interconnections between the two spheres of economic activity. David Lancashire [6], for example, employs a political economy perspective to explain the distribution of F/OS development worldwide. At the same time large scale surveys of F/OS communities indicate that approximately half of F/OS developers have earned money through their work in F/OS [7].

Although there has been considerable progress in understanding the links between the exchange economy and the gift economy at the level of developer motives, few studies have examined in detail how commercialization affects cooperation within projects. O'Mahony [1] approached the issue by focusing on the organizational structures that projects develop in order to interface with companies and West and O'Mahony [8] contrasted the dynamics of cooperation between community led and sponsored projects. Freeman and Siltala [9] have highlighted the hybrid practices developing in GNOME and Open Office due to corporate involvement. Adopting a different perspective Lin [10] examined developers' practices in firms that participate in F/OS development and the forms and tensions that arise from this collaboration. In addition, Dahlander and Magnusson [11] have examined the types of relationships companies develop with communities. Lastly, Krishnamurti and Tripathi's [12] study of bounty programs that offer developers monetary incentives for addressing specific 
software issues, highlights an alternative used by companies to participate in the development process rather than directly hiring developers.

This paper complements these contributions by developing a framework for understanding the dynamics of cooperation between corporate actors and volunteers through the involvement of paid developers. The theoretical and methodological framework for the research reported here is outlined in the next section together with highlights of the empirical data and the method of analysis.

\subsection{Theoretical framework, empirical data and method of analysis}

The study draws on the communities of practice perspective [13] and mobilizes Foucault's idea of relational power [14] in order to study relations between the volunteer community and commercial actors with stakes in the development.

The communities of practice perspective argues that society's knowledge is organized in different communities of practice, which are essential groups formed around the pursuit of a shared enterprise[15]. The approach offers an intuitive way of understanding F/OS development (since F/OS communities are essentially built around the practice of developing software) and has been adopted within the context of other investigations of F/OS [16-18]. In the context of this paper it is argued that F/OS communities do not constitute a homogenous community of practice consisting only of experienced and new (peripheral) developers, but form constellations of practices [13].

Foucault argued that power is neither a zero-sum game where different actors compete for resources nor something that is given or exchanged, but rather is something that is exercised; a force that creates complex dependencies and invites a diversity of initiations and reactions on the part of the people involved in them. The idea of relational power does not imply that the relations to be examined are symmetrical, but is meant to acknowledge and map the multiple interdependencies and structures that are developed within the context of this study.

Both these approaches have methodological implications. The idea of F/OS communities as constellations of practice draws attention to the different groups of contributors operating within projects, groups with distinctive characteristics and modes of engagement (volunteers, paid developers external to the community, community integrated developers). At the same time, the idea of relational power guides an investigation that aims to highlight the interdependencies forming between the different groups of contributors at different levels of interaction.

The data presented in this paper are drawn from 40 individual, semi-structured interviews conducted with volunteer and paid contributors from the GNOME and $\mathrm{KDE}$ projects between 2004-2005. Their analysis involved a two-step process. First the materials were organized thematically and then were analysed in more depth using a form of discourse analysis. This involved the identification of the particular domains of reference, types of calculation and forms of statement connected with specific knowledge domains, and the examination of the associations made between 
them [19] (F/OS, traditional working environments, volunteerism, professionalism, etc).

\section{Research findings}

Based on the analysis of the empirical data, a typology for understanding sponsorship and employment in community led F/OS projects is developed (section 3.1) followed by a consideration of the dynamics of cooperation developing between volunteer and paid developers based on the latters' community ties.

\subsection{Types of employment and sponsorship}

From the analysis of the interview data it seems that, depending on the kind of mandate they are given, paid developers can be divided in the following categories:

a. Those that have a free sponsorship. This group receives no clear instruction from their employers about what they should be working on. They are usually former volunteers who are expected more or less to work on the same things that they were contributing to before they were hired.

b. Those that have a clear mandate from their employers as to what they should be doing. For example, some developers working at Linux distribution companies, are expected to help integrate different aspects of the project into company products or build on top of their projects' platforms to create commercial applications.

c. Those that are have KDE or GNOME "friendly" jobs. These are people who are hired by companies or departments of companies with a strong F/OS orientation. They are usually hackers who are expected to work on developing proprietary company products, but who are also allowed to spend part of their time working on community projects. Their working terms, can be formal or informal, and resemble part-time free sponsorship.

d. Those that are being hired or compensated in order to solve a particular problem or develop a specific application. This type of involvement is akin to subcontracting. For example, Sun Microsystems, a company involved in Gnome development has hired developers from Wipro, a large Indian software house, to help them on certain aspects that their own team did not have the time to deal with. This category could also include development conducted within the context of bounty programs, and self-employed developers.

\subsection{Working from the outside and the inside: external and community integrated contributors and community development}

In addition to the above categorization, there is an important distinction to be made between contributors that formerly worked for the project on a volunteer basis and those external programmers who are commissioned to work on it without having any 
previous ties with the community. Proprietary developers that are brought to work on community projects have to learn the ways of the community and adjust to the rhythms and the demands of F/OS development. Most interviewees tended to think that this group has significant difficulties in adjusting to the often unstructured work flows of the community.

Some of these problems have been resolved. The regularization of release cycles for GNOME and KDE, for example, allows companies to better time the release of their own products. Although they stressed the frequent incommensurability of community and corporate agendas and processes (which was often seen as a divide between the F/OS way and proprietary development) almost all the interviewees believed that these days communities are doing more to accommodate companies' needs. Despite the progress being made, however, there appear to be more gaps than areas of contact in the more formal aspects of community-corporate cooperation, such as the incommensurability between companies' Quality Assurance processes and those of the community (such as its bug-tracking systems).

On the community's part companies' contributions to the code base can be too specialized for the overall aims of the project and, consequently, the issue of "pushing the patches upstream" (i.e. integrating them into the main development tree) does not make any sense, or they may be relevant. In the latter case companies usually pursue integration because it saves on the time and effort required to maintain the changes made to the code base. An underlying assumption in the acceptance of code submitted by paid contributors is that it is done on the same kind of meritocratic basis that applies to the rest of the community.

Some of the issues are aptly illustrated by the case of Wipro. Wipro, an Indian Software House, was subcontracted by Sun Microsystems to work on the GNOME project. Although they were expected to work with the community, Wipro developers were not expected to become members. Their success was measured according to specific metrics (bugs fixed, bugs logged). Due, however, to their lack of prior knowledge about the code base and to the inadequacy of available documentation they had to resort to asking for information on community mailing lists and chat channels. The presence of some 10 new developers all asking questions stretched the learning resources of the community and created a stir among volunteer developers.

Developers that worked as volunteers or paid developers who became accepted as community members based on their longstanding contributions, carry with them a network of connections and an extensive know-how of community processes that both facilitates their work in terms of its acceptance by the community and helps connect corporate and community teams.

In companies such as Sun Microsystems that have large teams of developers working on F/OS projects, these people sometimes assume the role of intermediary between the rest of the corporate team and the community.

A company's strategy to recruit volunteers who are already on a "critical path"1 in the project, who are heavily involved in central aspects of development, means that very often employed contributors are maintainers of key parts of the project. It is a

\footnotetext{
I I would like to thank Luis Villa for suggesting this term.
} 
plausible hypothesis that the combination of their potential as volunteer contributors with the opportunity to work full-time in the project enhances their position as core developers. These central actors not only have the ability to control key aspects of the project, but also have an interest in keeping an eye on its overall development.

At the same time employed community members demonstrate an increased sensitivity to community issues, which makes them cautious with regard to balancing community and company interests. Employers' links with projects and with the F/OS source community in general affects developers' relations with their employer and influences their everyday working life. Where companies have strong ties with the F/OS world the confluence between community and corporate interests appears relatively uncomplicated. The fact that they do not need to explain community processes and policies makes the developers' relationship with the management easier. More importantly, in contrast to working in a company with a less developed F/OS culture, upholding community values and ideals seems to be conducted not in opposition (we and the rest of the company) but collectively (we with the rest of the company in F/OS).

\section{Conclusions}

This paper elaborated an initial framework for understanding the role of paid developers in community led F/OS projects. After developing a typology of their employment/sponsorship arrangements (free sponsorship, clear mandate, F/OSfriendly jobs, subcontracting) the differing community ties of employed developers were examined.

The role of developers with weak community ties, those external to the community, is consistent with the view of companies as external actors in the development process, whose involvement in the project is regulated through community values and mediated through appropriately developed institutional interfaces. The case of community-integrated employees, however, suggests that the boundaries between corporate and community actors are often permeable. More specifically, it appears that in most cases the connections between companies and projects develop at different levels of involvement and hinge upon complex interpersonal dynamics. Many of the elected members on the Foundation and the KDE e.V. Boards for example, are either hired or self-employed contributors.

It should be noted that despite their involvement in projects, companies generally avoid exercising direct pressures on the community, since it is in their interests that projects retain their largely volunteer basis. It appears that the preservation of the balance in favour of the gift economy is as much to the benefit of companies as is that of communities.

The analysis in this paper is a first attempt to untangle some of the complex issues underlying community-corporate cooperation in F/OS projects. One of the most important emerging questions is whether paid developers constitute a distinctive group, not just at the level of engagement with the community, but at the level of their 
contributions. The interview data suggest that the group of community integrated employed developers includes members of the group of core developers, programmers who contribute to the programs' most critical parts. This question is addressed through examining the findings of a social network analysis of the Gnome's Foundation and KDE e.V. members (see [20]). The question of how employment/sponsorship arrangements affect cooperation between paid and volunteer developers and how community and corporate boundaries are managed within the context of development is not addressed in this paper.

Despite its limitations the paper proposes a framework for understanding the complex interdependencies that frequently develop between companies and communities not only at the institutional level, but within the context of mundane development. In addition it offers a view of F/OS projects not as uniform communities organized around a homogenous practice, but as complex spaces incorporating different types of contributors. In doing so it invites an examination not just of the differences and barriers existing between the exchange and the gift economy, but of the ways they are embedded in each other.

The research was funded by the Greek State Scholarships Foundation (IKY). In its later stages the study was supported by the EU Digital Business Ecosystem (DBE) project (contract no 507953) http://www.digital-ecosystem.org/. The views expressed in this paper are those of the author.

\section{References}

1. C. S. O'Mahony, 'The emergence of a new commercial actor: community managed software projects.' Doctoral Dissertation, Stanford University, Department of Management Science and Engineering Management, (2002).

2. E. von Hippel, Open Source Projects as Horizontal Innovation Networks-by and for users. MIT Sloan School of Management, Working Paper No. 4366-02, Boston, MA 2002 (accessed 23/07/2003).

3. M. S. Elliot and W. Scacchi, Free Software: a Case Study of Sofware Development in a Virtual Organization Culture. Working Paper, Institute for Software Research, UC Irvine, April 2003, available at: http://www.ics.uci.edu/\%7Ewscacchi/Papers/New/Elliott-Scacchi-GNUe-Study-

Report.pdf 2003 (accessed 6/06/04).

4. K. Lakhami and R. G. Wolf, Why hackers do what they do: Understanding Motivation Effort in Free/Open Source Software Projects. MIT Sloan School of Management Working Paper No. 4425-03, 2003 (accessed 01/03/04).

5. E. S. Raymond, The Cathedral and the Bazaar. The Cathedral and the Bazaar: Musings on Linux and Open Source by an Accidental Revolutionary, ed. E. S. Raymond (O'Reilly, Sebastopol, CA, 2001MC).

6. D. Lancashire, Code, Culture and Cash: The Fading Altruism of Open Source Development, First Monday, peer-reviewed journal on the Internet 6(12) (2001). 
7. P. David, A. Waterman and S. Arora, FLOSS-US/ The Free/Libre/Open Source Developer Software Survey for 2003: A First Report. http://www.stanford.edu/group/floss-us/report/FLOSS-US-Report.pdf, 2003 (accessed 5/5/04).

8. J. West and C. S. O'Mahony, Contrasting Community Building in Sponsored and Community Founded Open Source Projects. http://opensource.mit.edu/papers/westomahony.pdf, 2004 (accessed 25/07/04).

9. S. Freeman and J. Siltala, Freedom and Profit: how suits and hackers are working it out on the desktop. Working paper presented in 4/EASST Joint Meeting, Paris 26/08/04 http://personal.inet.fi/koti/jsiltala/juha/floss/freedom-profit-paper.pdf, 2004 (accessed 19/01/06).

10. Y. Lin, Hybrid Innovation: How Does the Collaboration Between the FLOSS Community and Corporations Happen?, Forthcoming in Knowledge, Technology and Policy XVIV (1) (2005).

11. L. Dahlander and G. M. Magnusson, Relationships between open source software companies and communities: Observations from Nordic Firms, Research Policy,(34): p. $481-493$ (2005).

12. S. Krishmamurti and A. Tripathi, Bounty Programs in Free/Libre/Open source Software (FLOSS): An Economic Analysis", in The Economics of Open Source Software Development (Forthcoming), J. Bitzer and P. Schroeder, Editors (Elsevier Publications, 2005).

13. E. Wenger, Communities of Practice: Learning, Meaning and Identity (Cambridge University Press, Cambridge, 1998).

14. M. Foucault, The Subject and Power, in Michel Foucault: Power/ Essential works of Foucault 1954-1984. Vol. 3, J. D. Faubion, Editor (Penguin Books, London; New York, 1982) p. 326-348.

15. E. Wenger and J. Lave, Situated learning: Legitimate Peripheral Participation (Cambridge University Press, Cambridge, 1991).

16. K. Edwards, Epistemic Communities,' Situated Learning and Open Source Software Development. http//opensource.mit.edu/papers/kasperedwards-ec.pdf, 2001 (accessed 1/03/05).

17. S. Sharma, V. Suguraman and B. Rajagopalani, A framework for creating hybrid open source communities, Information Systems Journal. 12: p. 7-25 (2002).

18. M. S. Elliot and W. Scacchi, Free Software Developers as an Occupational Community: Resolving Conflicts and Fostering Collaboration. In revised version in ACM Group'03 Conference. 2003. Sanibel Island, FL 9-12.

19. G. Kendall and G. Wickham, Using Foucault's methods (Sage, London, 1998).

20. E. Berdou, 'Commercialization and Peripheral Participation in Community led Free/Open Source Projects: Evolving Forms of Work and Power at the Intersection of Online and Offline Worlds.' Doctoral Dissertation, London School of Economics and Political Science, Media and Communications Department, (Forthcoming 2006). 